\title{
SQUID-Detected in Vivo MRI at Microtesla Magnetic Fields
}

\author{
Michael Mößle, Whittier R. Myers, SeungKyun Lee, Nathan Kelso, Michael Hatridge, Alexander \\ Pines and John Clarke
}

\begin{abstract}
We use a low transition temperature $\left(T_{c}\right)$ Superconducting Quantum Interference Device (SQUID) to perform in vivo magnetic resonance imaging (MRI) at magnetic fields around 100 microtesla, corresponding to proton Larmor frequencies of about $5 \mathrm{kHz}$. In such low fields, broadening of the nuclear magnetic resonance lines due to inhomogeneous magnetic fields and susceptibility variations of the sample are minimized, enabling us to obtain high quality images. To reduce environmental noise the signal is detected by a second-order gradiometer, coupled to the SQUID, and the experiment is surrounded by a 3-mm thick Al shield. To increase the signal-tonoise ratio (SNR), we prepolarize the samples in a field up to 100 $m T$. Three-dimensional images are acquired in less than 6 minutes with a standard spin-echo phase-encoding sequence. Using encoding gradients of $\sim 100 \mu \mathrm{T} / \mathrm{m}$ we obtain threedimensional images of bell peppers with a resolution of $2 \times 2 \times 8$ $\mathrm{mm}^{3}$. Our system is ideally suited to acquiring images of small, peripheral parts of the human body such as hands and arms. In vivo images of an arm, acquired at $132 \mu \mathrm{T}$, show 24-mm sections of the forearm with a resolution of $3 \times 3 \mathrm{~mm}^{2}$ and a SNR of 10 . We discuss possible applications of MRI at these low magnetic fields.
\end{abstract}

Index Terms - In vivo images, magnetic resonance imaging, nuclear magnetic resonance, SQUID

\section{INTRODUCTION}

$\mathrm{I}^{\mathrm{n}}$ $n$ magnetic resonance imaging (MRI), a spatial map of the nuclear magnetic resonance (NMR)-active nuclei is obtained by detecting the magnetic signal from nuclear spins precessing in the presence of a magnetic field gradient [1], [2]. In addition to the image encoding gradients, traditional MRI utilizes a strong, uniform field $B_{0}$ for two purposes: to polarize

Manuscript received October 4, 2004. This work was supported by the Director, Office of Science, Office of Basic Energy Sciences, Division of Materials Sciences and Engineering of the U.S. Department of Energy under Contract No. DE-AC03-76SF00098.

M. Mößle is with the Physics Department, University of California and the Materials Sciences Division, Lawrence Berkeley National Laboratory, Berkeley, CA 94720 USA (phone: 510-642-3634; fax: 510-642-1304; e-mail: mjmoessle@lbl.gov).

W. R. Myers (email: wmyers@socrates.berkeley.edu), S-K. Lee (email: 1sk@socrates.berkeley.edu), N. Kelso (email: kelso@socrates.berkeley.edu), M. Hatridge (email: hatridge@berkeley.edu) and John Clarke (email: jclarke@socrates.berkeley.edu) are with UC Berkeley and LBNL, Berkeley, CA 94710, USA.

A. Pines is with the Chemistry Department, UC Berkeley and the Materials Sciences Division, LBNL, Berkeley, CA 94720, USA (email: pines@berkeley.edu). the spins in the sample and to achieve a spin precession frequency of tens to hundreds of $\mathrm{MHz}$. The spins precess at a frequency $f_{0}=\gamma B_{0} / 2 \pi$, and the resulting signal is detected by Faraday induction; $\gamma$ is the gyromagnetic ratio. For ${ }^{1} \mathrm{H}$, $\gamma / 2 \pi=42.6 \mathrm{MHz} / \mathrm{T}$. Because the signal from Faraday induction scales as the precession frequency and the spin polarization increases linearly with $B_{0}$, in conventional MRI the detected signal scales as $B_{0}{ }^{2}$. Thus there is great incentive to operate at high magnetic fields, and $B_{0}$ typically ranges from 1-4 $\mathrm{T}$ in clinical imaging.

Since the early 1990s, however, an alternative approach of polarizing the spins with a pulsed field $B_{p}$ and detecting them in a much lower field has attracted interest [3], [4]. This approach has a number of advantages. First, because resolution limitations caused by field inhomogeneity scale with the magnitude of $B_{0}$, the relative homogeneity requirement of $B_{0}$ is much relaxed by reducing $B_{0}$. Since $B_{p}$ does not require high homogeneity, one can use a weak, moderately homogenous $B_{0}$ field and a strong, inhomogeneous $B_{p}$ field; both fields can be generated from copper wire coils and do not require shimming. Second, when one encodes images in a low field $(<0.1 \mathrm{~T})$ artifacts caused by susceptibility changes in the sample and by chemical shift are largely eliminated. Finally, in contrast to the case for superconducting magnets, the field from resistive magnets can be easily varied, enabling the measurement of NMR properties of the sample over a wide range of fields. In particular, image contrast caused by differences in the spin-lattice relaxation time $\left(T_{1}\right)$ is often enhanced at low magnetic fields [5], [6].

To avoid the sensitivity loss inherent in low-frequency Faraday detection, one can use a SQUID detector [7]-[9]. Coupled to a superconducting flux transformer, a SQUID can have a field sensitivity of $\sim 1 \mathrm{fT} \mathrm{Hz}^{-1 / 2}$ at frequencies ranging from several megahertz down to a few hertz [10]. Combining SQUID detection with prepolarization allows frequencyindependent MRI detection.

We used this combined approach of SQUID detection and prepolarization with low-field encoding in our earlier work $[11,12]$ to acquire two-dimensional images of water and oil phantoms and of vegetables with 2-4 $\mathrm{mm}$ spatial resolution in an encoding field of $132 \mu \mathrm{T}$. In this paper, we employ a three-dimensional phase-encoded pulse sequence to produce three-dimensional in vivo images of human arms and fingers in microtesla fields. 


\section{EXPERIMENT}

\section{A. SQUID detector and magnetic field coils}

The measurement principle of SQUID-detected MRI is shown schematically in Fig. 1 (a). The magnetic field from proton spins precessing around the measurement field $B_{0}$ is detected by the lowest coil of a $\mathrm{Nb}$-wire, second-order axial gradiometer with $1+2+1$ turns. The baseline is $75 \mathrm{~mm}$ and the loop diameters are $65 \mathrm{~mm}$. It has a balance of $\sim 100$ against uniform fields, thereby reducing external noise from distant sources. The gradiometer is connected to a multiturn input coil of a thin-film, low- $T_{c}$ SQUID [Quantum Design, San Diego] based on $\mathrm{Nb}-\mathrm{AlOx}-\mathrm{Nb}$ tunnel junctions. The effective area of a single loop of the gradiometer is $\sim 7 \mathrm{~mm}^{2}$. The SQUID is operated in a flux-locked loop with flux modulation at $2 \mathrm{MHz}$. The measured flux noise in the flux-locked loop is $6 \mu \Phi_{0} \mathrm{~Hz}^{-}$ ${ }^{1 / 2}$, yielding a magnetic field noise of $1.7 \mathrm{fT} \mathrm{Hz}^{-1 / 2}$ relative to the bottom loop of the gradiometer. In order to protect the pickup coil from high currents in the input circuit during the polarizing pulse, an array of Josephson tunnel junctions is inserted into the input circuit. The normal resistance of this array is $\sim 800 \Omega$.

The SQUID and gradiometer are mounted in a low-noise cryostat; the sample is placed beneath the cryostat, at a distance of $25 \mathrm{~mm}$ below the bottom loop of the gradiometer. To minimize the noise contribution from the thermal shielding of the cryostat we employ a G-10 fiberglass dewar with superinsulation consisting of aluminized cloth [13]. The construction of this dewar is described in detail elsewhere [12]. The measured magnetic field noise of this cryostat is well below the intrinsic noise level of the SQUID.

Fig. 1 (b) shows a schematic of the magnetic field and gradient coils. Three orthogonal pairs of coils on a $1.8 \mathrm{~m}$ wooden cube are used to cancel the earth's magnetic field. The precession field $B_{0}(60$ to $130 \mu \mathrm{T})$ is provided by a Helmholtz pair of coils. Longitudinal $\left(G_{z}\right)$ and transverse $\left(G_{x}\right.$ and $G_{y}$ ) field gradients are applied by sets of coils, wound in the Maxwell and Golay geometries; specifications for all coils used in this experiment can be found elsewhere [12]. The wooden cube also supports the cryostat. The polarizing coil is mounted 10 to $70 \mathrm{~mm}$ below the cryostat and samples are placed in the gap between the cryostat and the polarizing coil. To reduce the additional noise created by Nyquist currents in the polarizing coil, we wound this coil out of 30-strand braided copper wire instead of solid wire. A pair of coils placed around the sample space applies the audiofrequency excitation pulses. To attenuate external noise, the entire experiment is enclosed in 3-mm thick Al plate. This shielding provides two skin depths of attenuation at $5.6 \mathrm{kHz}$, the NMR frequency at $132 \mu \mathrm{T}$.
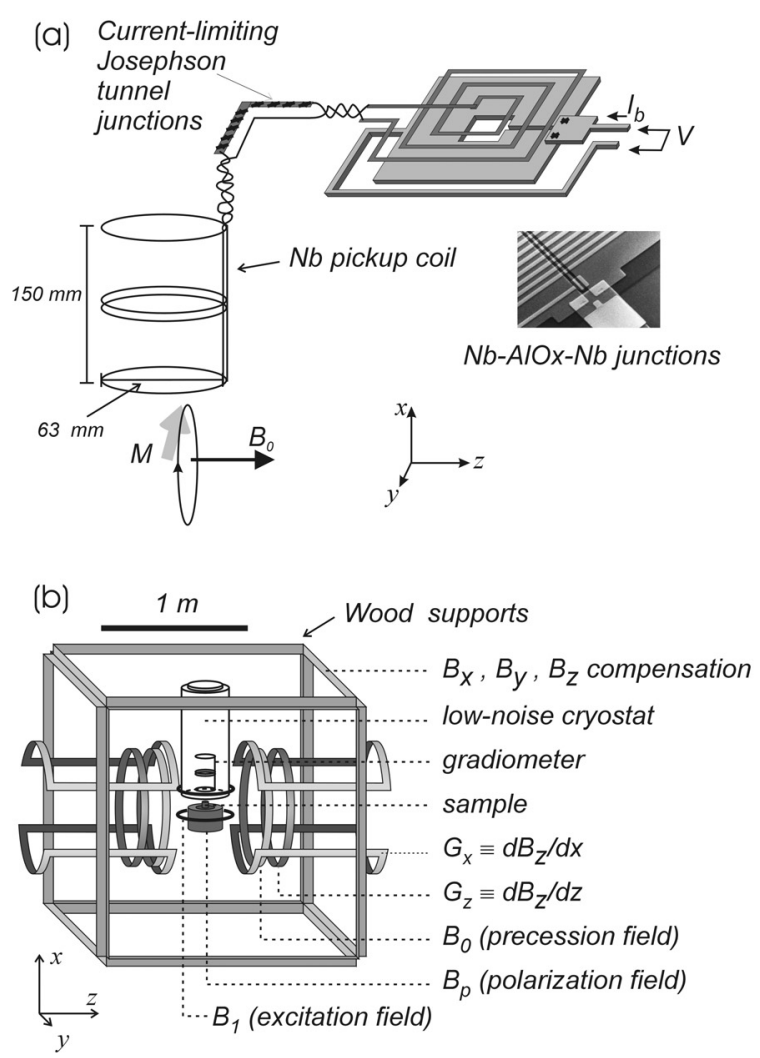

Fig. 1. (a) Measurement principle for SQUID-detected MRI. (b) Configuration of the SQUID MRI system showing magnetic field and gradient coils and the liquid helium dewar; for clarity $G_{y}$ coils are not shown.

\section{B. Pulse Sequence}

To obtain two- and three-dimensional images of our samples we used the pulse sequence shown in Fig. 2. First the spins were prepolarized in a magnetic field $B_{p}$ between $40 \mathrm{mT}$ and $100 \mathrm{mT}$ along the $x$-direction for a time comparable to $T_{1}$. This field was then turned off adiabatically, causing the spins to align along the much weaker precession field $B_{0}$. After a delay time $t_{d}$ a resonant $90^{\circ}$ pulse induced spin precession around $B_{0}$. At a time $\tau$ later, a resonant $180^{\circ}$ pulse was applied to form a spin-echo signal. This signal was detected by the SQUID, digitized by an analog-to-digital converter and stored in a desktop computer. Two-dimensional spatial encoding was achieved with a standard phase-encoding sequence [14] incorporating a constant frequency-encoding gradient and a phase-encoding gradient applied only between the $90^{\circ}$ and $180^{\circ}$ pulses. Spatial encoding in the third dimension was performed by adding a second phase-encoding gradient. The full three-dimensional image was obtained by a threedimensional fast Fourier transform of the softwaredemodulated data. For our prepolarized SQUID-detected MRI experiment this 3D-pulse sequence gives better SNR than a comparable sequence that employs slice-selection along the third dimension [15]. 


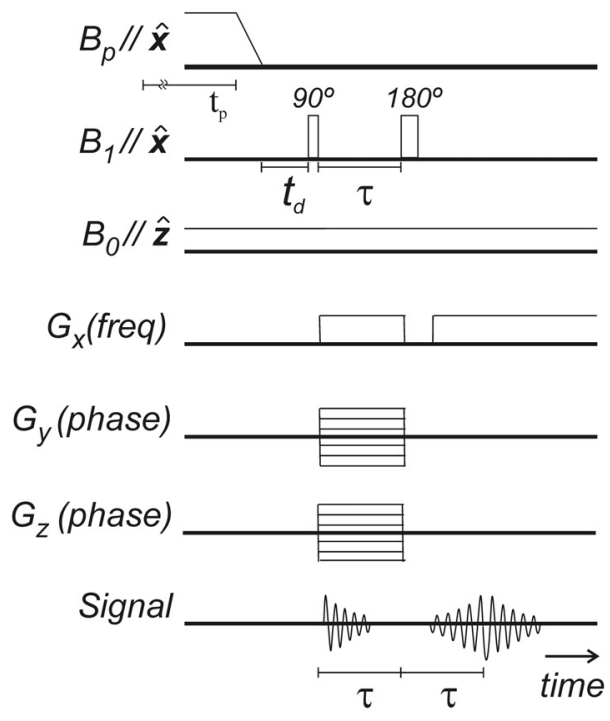

Fig. 2. Pulse Sequence for three-dimensional imaging.

\section{RESUlTS AND DisCUSSION}

\section{A. Three-dimensional images of bell peppers}

To test the performance of the system and to adjust the pulse sequence, we first acquired three-dimensional images of bell peppers. The pepper, oriented along the $y$-direction, was placed horizontally between the bottom of the dewar and the polarizing coil. The average polarizing field was $40 \mathrm{mT}$, applied for $500 \mathrm{~ms}$, and the measurement field was $66 \mu \mathrm{T}$. The images in Fig. 3 were taken in 5 min with a frequencyencoding gradient of $57 \mu \mathrm{T} / \mathrm{m}$ along the $x$-direction, 31 phaseencoding steps along the $y$-direction and 9 phase-encoding steps along the $z$-direction. The maximum phase-encoding gradient was $100 \mu \mathrm{T} / \mathrm{m}$ in both directions. Fig. 3 (a) shows six cross sections of the pepper. The thickness of each section is 8 $\mathrm{mm}$, the SNR is 10 and the in-plane resolution is $2 \mathrm{~mm} \times 2$ $\mathrm{mm}$. All images show a significant decay in signal along the $x$-direction due to the increasing distance from the gradiometer.

Fig. 3 (b) shows a three-dimensional image of the same pepper, enclosed in a $200 \mu \mathrm{m}$ thick aluminum can, using the same pulse sequence. At $2.8 \mathrm{kHz}$, the measurement frequency used for this image, the skin depth of aluminum is $\sim 2 \mathrm{~mm}$, so that the excitation pulses are not significantly attenuated by the metal surrounding the sample. In contrast, at the $64 \mathrm{MHz}$ frequency used in conventional $1.5 \mathrm{~T}$ MRI scanners the skin depth of the metal would be $\sim 20 \mu \mathrm{m}$, so that the metal would screen radiofrequency pulses applied to the sample as well as the signal from the sample. Furthermore, eddy currents induced in the metal would greatly distort the image, thereby limiting the resolution. Our images of samples inside the can show no decrease in signal compared to measurements of the sample taken without the can. However, Nyquist currents in the aluminum increase the noise level, so that the overall SNR of the images is lowered. In the image shown in Fig. 3 (b) the number of averages was increased from 3 to 4 to achieve the same SNR and resolution as in Fig. 3 (a). No distortion of the image due to the proximity of the metal is visible. Those results show that imaging samples surrounded by metal or containing metal is possible at the measurement frequencies

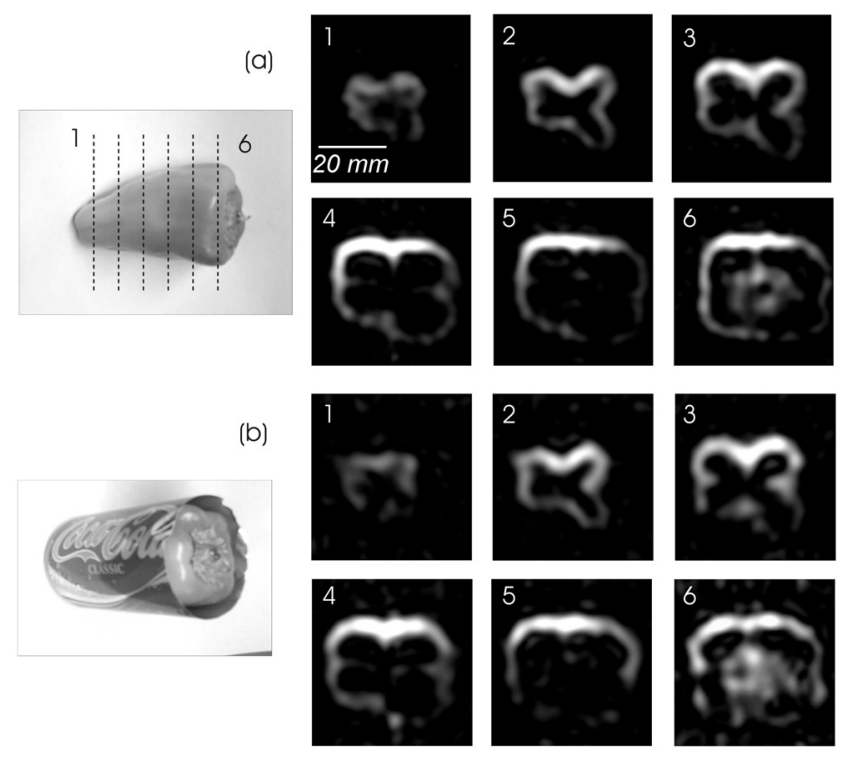

used in our system.

Fig. 3. (a) Three-dimensional image of bell pepper showing six cross sections with a thickness of $8 \mathrm{~mm}$; the lines in the photograph mark the position of each slice. (b) Six cross sections of the same pepper enclosed in an aluminum can.

\section{B. In vivo images}

In its present configuration the system is ideally suited to acquire in vivo images of small, peripheral parts of the human body such as hands and arms. To acquire an image, the hand or arm is placed in the 70-mm gap between the bottom of the dewar and the polarizing coil, surrounded by the two halves of the excitation coil. Three-dimensional images of a a human forearm were acquired at $B_{0}=132 \mu \mathrm{T}$ with an average prepolarization field $B_{p}=40 \mathrm{mT}$. Figs. 4 (a) and (b) show two 24-mm thick sections of the forearm with a SNR of 10 and an in-plane resolution of $3 \mathrm{~mm} \times 3 \mathrm{~mm}$; the imaging time was 6 minutes. In these images the bone marrow of the radius and ulna and the subcutaneous fat layer appear bright. Most other parts of the arm, including the muscle, appear dark. This tissue contrast is caused by transverse relaxation time $\left(T_{2}\right)$ weighting. After the $90^{\circ}$ pulse, the spins dephase with time constant $T_{2}$. For fatty tissue and yellow bone marrow, $T_{2}$ ranges from $60-100 \mathrm{~ms}$, whereas for muscle $T_{2} \sim 20 \mathrm{~ms}$. In these images, the spin echo occurs $35 \mathrm{~ms}$ after the $90^{\circ}$ pulse; at this point the spins in the muscle are almost completely dephased and do not contribute to the image. As in Fig. 3, all images show a decay in signal along the $x$-direction due to the increasing distance from the bottom loop of the gradiometer. Fig. 4 (c) shows the same slice as in Fig. 4 (b) after dividing by the modeled response function of the pickup coil. 

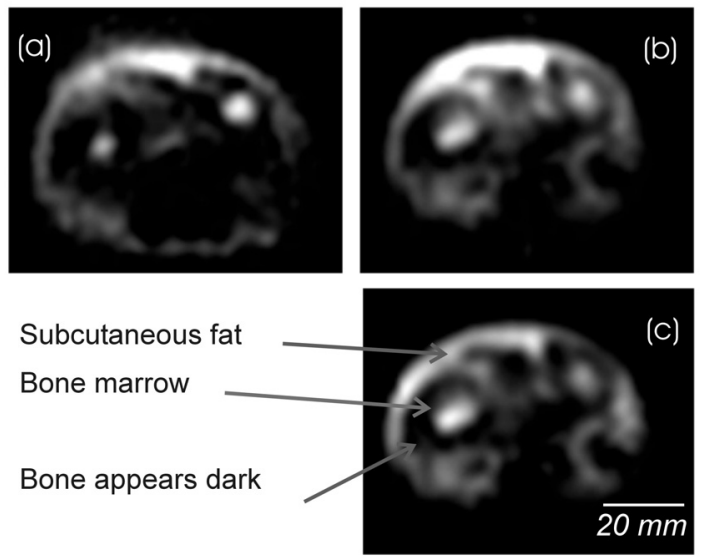

Fig. 4. (a), (b) Two cross sections at different positions of a forearm; thickness of one slice is $24 \mathrm{~mm}$. (c) Same image as (b) with amplitude correction.

Another example of in vivo images taken with our system is shown in Fig. 5. These images show four different cross sections of human fingers. The fingers were placed in the 30$\mathrm{mm}$ gap between the bottom of the dewar and the polarizing coil; this smaller separation allows us to apply a larger average polarization field of $60 \mathrm{mT}$. Here the same $T_{2^{-}}$ weighted pulse sequence with $2 \tau=35 \mathrm{~ms}$ was used. As in the images of the forearm, bone marrow and subcutaneous fat appear bright, and all other tissues appear dark. The thickness of one slice is $12 \mathrm{~mm}$, the imaging time is 6 minutes, the signal-to-noise ratio is about 10 and the in-plane resolution is better than $2 \times 2 \mathrm{~mm}^{2}$.

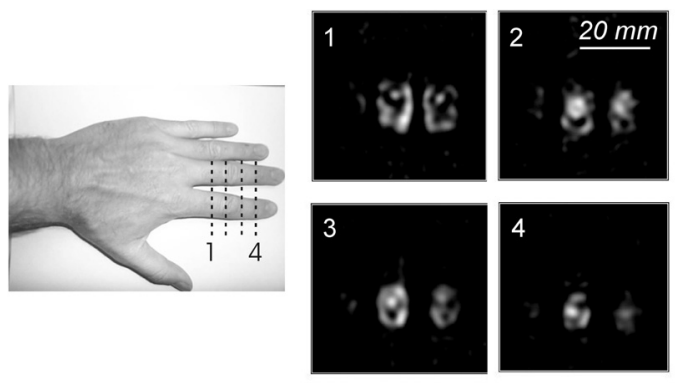

Fig. 5. $12 \mathrm{~mm}$-thick cross sections of human fingers; position of each slice is marked on the photograph of the hand.

\section{CONCLUSIONS}

By prepolarizing the spins and detecting the spin precession at 2.8 to $5.6 \mathrm{kHz}$ with an untuned SQUID gradiometer, we obtained three-dimensional images of bell peppers and of human arms and fingers with 2-3 mm in-plane resolution and imaging times of a few minutes. To our knowledge, these images of the arm and fingers are the first in vivo human images obtained in a microtesla magnetic field by a SQUID sensor. The image of the pepper inside an aluminum can shows no appreciable distortion due to the proximity of the metal and suggests that imaging of patients with metallic implants or fillings is a possibility with SQUID-detected microtesla-field MRI.

Currently, image resolution and SNR are mainly limited by the magnetic field noise of the SQUID gradiometer and the available polarizing field strength. By using a SQUID with a lower flux noise and increasing the effective area of the gradiometer, we can reduce the present detector noise of 1.7 fT $\mathrm{Hz}^{-1 / 2}$ by nearly an order of magnitude. A corresponding reduction in the enviromental field noise could be achieved by improving the balance of the gradiometer. Furthermore, by suitable design and cooling of the polarizing coil one could increase $B_{\mathrm{p}}$ to $150 \mathrm{mT}$ or higher in a system dedicated to specific applications, for example, imaging the wrist. With these improvements in SNR and resolution, SQUID-detected microtesla in vivo MRI may well find novel applications in specialized clinical imaging of human subjects, for example, low-cost tumor screening using low field relaxation properties of normal and malignant human tissues, and measuring the longitudinal relaxation time of bone marrow.

\section{ACKNOWLEDGMENT}

We thank R. McDermott, A. H. Trabesinger, S. Han, and B. Inglis for helpful discussions, and $\mathrm{H}$. Seton for providing superinsulation and his plans for a low-noise dewar. M. Mößle was supported by the "Deutsche Forschungsgemeinschaft" through a postdoctoral fellowship.

\section{REFERENCES}

[1] P. C. Lauterbur, "Image formation by induced local interactions Examples employing nuclear magnetic resonance," Nature, vol. 242, pp. 190-191, 1973.

[2] P. Mansfield and P. K. Granell, "NMR diffraction in solids?," J. Phys. C, Solid State Phys., vol. 6, pp. L422-L426, 1973.

[3] A. Macovski and S. Conolly, "Novel approaches to low cost MRI," Magn. Reson. Med., vol. 30, pp. 221-230, 1993.

[4] G. Planinšič, J. Stepišnik, and M. Kos, "Relaxation-time measurement and imaging in the earth's magnetic field," J. Magn. Reson. Ser. A, vol. 110, pp. 170-174, 1994.

[5] S. H. Koenig and R. D. Brown III., "Relaxometry of Tissue," in NMR Spectroscopy of Cells and Organisms, vol. 2, R. K. Gupta, editor, Boca Raton FL: CRC Press, 1987, pp. 75-114.

[6] S-K. Lee et al. "SQUID-Detected MRI at $132 \mu \mathrm{T}$ with $T_{1}$ contrast weighted at $10 \mu \mathrm{T}-300 \mathrm{mT}$," Magn. Reson. Med., to be published.

[7] M. P. Augustine et al., "Low field magnetic resonance images of polarized noble gases obtained with a dc superconducting quantum interference device," Appl. Phys. Lett., vol .72, pp. 1908-1910, 1998.

[8] H. C. Seton, J. M. S. Hutchinson, and D. M. Bussel, "A 4.2 K receiver coil and SQUID amplifier used to improve the SNR of low-field magnetic resonance images of of the human arm," Meas. Sci. Tech.," vol. 8, pp. 198-207, 1997.

[9] A. N. Matlachov et al., "SQUID detected NMR in microtesla magnetic fields," J. of Magn. Reson., vol. 170, pp. 1-4, 2004.

[10] R. Kleiner, D. Koelle, F. Ludwig, and John Clarke, "Superconducting quantum interference devices: state of the art and applications," Proceedings of the IEEE, vol. 92, pp. 1534-1548, 2004.

[11] R. McDermott, S-K. Lee, B. ten Haken, A. H. Trabesinger, A. Pines, and J. Clarke, "Microtesla MRI with a superconducting quantum interference device," Proc. Nat. Acad. Sci. , vol. 101, pp. 7857-7861, 2004.

[12] R. McDermott et al., "SQUID-detected magnetic resonance imaging in microtesla magnetic fields," J. Low Temp. Phys., vol. 135, pp. 793-821, 2004.

[13] H. C. Seton, D. M. Bussell, and J. M. S. Hutchinson, United Kingdom Patents GB2331798, 2000 and GB2351549, 2001. 
[14] E. M. Haake, R. W. Brown, M. R. Thompson, and R. Venkatesan, Magnetic Resonance Imaging: Phyiscal principles and sequence design. New York: Wiley, 1999.

[15] Ref. [14], p. 347. 